\title{
III \\ DE CODICIBUS LIBRI DE FATO
}

$V^{1} \quad$ Etiam libellum, quem De Fato ad Imperatores misit, licuit ex VENETo Marciano 258 edere. neque differt optimi libri indoles in hac argumenti parte. inveniuntur et crebrae sub primae manus scriptura liturae et voculae breviores sive infra sive supra versum additae. neque desunt curae, qua vetustius exemplar deseripsit librarius, testimonia, verborum dico sive syllabarum in suas partes nondum discriptas conglobationes, quibus quippe haud intellectis prosodiam ille non adpinxit ef. 176,3.7.21 185,7.8.25 186,27 187,8 189,16 193,16.17 195,15 197,6 203,4.

Paucas ut in ceteris partibus invenit in archetypo varias lectiones $164,7167,14171,25$ ?, quod mirabere in libello omnium lectioni destinato. saepe inter scribendum substitisse manum primam iterumque examinasse quod scripserat hic quoque docent praeter lituras cum transposita verba $(186,1 \quad 196,16198,12199,22)$ tum hi loci 182,2 202,2. $12.13203,18$ ubi librarii sedulitatem adgnoscimus de litterarum in vetusto exemplo evanidarum significatione dubitantis (cf. p. XVI).

$\mathrm{V}^{2} \quad$ Pertractavere libellum et vetus corrector et manus altera. huius autem post tertiam ab initio partem vestigia non inveniuntur neque ea quae huic parti praestitit aliter censebimus, quam supra factum est. proclive enim erat bonum supplementum excogitare ad 169,20 et adgnoscimus felicem docti hominis manum emendatricem cum 165,1.2.10.12 166,18. 19 167,12. 21 169,31 170,3 171,2.5 (xаi) 172,11. 13. 15.25 173,24 175,18. 20176,7 tum 165,20 172,28. sed hic quoque, ut par est, haud raro erravit is, qui ingenii tantum subsidiis instructus erat, neque vituperabimus vana tentamenta, ut $164,20 \quad 166,23 \quad 168,6 \quad 169,24-25 \quad 171,4.5$ 172,14 . 15173,26 , immo laudabimus eum ad gravius corrupta manum non admovisse, ut $165,3166,28 \quad 170,25$. 
Per totum autem libellum veteris correctoris praenitent merita. v. c. V cui quid debeamus in nulla codicis regione magis elucet. primum igitur permulta correxit, of. 176,16 179,23.24 (థَ) 181,5 184,2 187,21.31 188,3.22

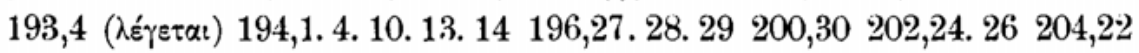
207,7. 8.28 210,18. tum plurimum profuit punctis adponendis, quibus in nulla codicis parte eopiosius usus est. ita primum singulis punctis singulas litteras delevit $169,24 \quad 172,915 \quad 177,13 \quad 179,6.10 \quad 180,10.15 \quad 184,24$ 186,24 194,24 195,19 203,26 204,10 205,2 206,19. 20 211,17 deinde singulas litteras suspectas esse indicavit 180,7.14 181,16 186,27 187,2 porro singulis syllabis fidem derogavit 181,14 182,16 187,12 188,11 $196,14204,3$, vocem ut falsam damnavit 186,19 delevit 176,23 . prae-

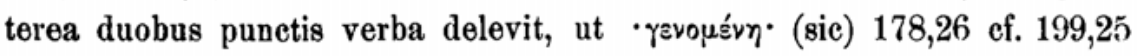

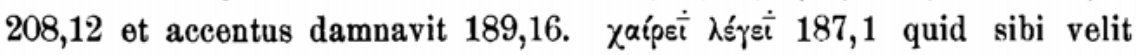
mihi non plane liquet. nam si lineola supraposita vice fungitur litterae $v$, cur puncto littera ut corrupta notetur non patet. fortasse igitur hoc ita accipiendum est, ut lectiones puncto designatae 173,4 192,15 195,6 196,2 200,26. his enim locis suis propositis punctum addidit vetus corrector, quo fortasse se ipsum dubitare fatetur. denique sunt, ubi signi consilium lateat: $166,5 \quad 173,27 \quad 175,7 \quad 180,21 \quad 181,25$ et erravit ar$\begin{array}{llllllll}\text { chetypi vetustate deceptus } & 173,5 & 179,23 & 182,32 & 186,27 & 193,4 & 196,23\end{array}$ 198,25 .

Praeter Venetum mihi praesto fuerunt hi codices apographi, chartacei omnes, saeculo XV-XVI. scripti

Venetus Marcianus 261 (cf. p. XIX) B

Venetus Marcianus App. cl. IV cod. 10 (cf. p. XX) S

Mutinensis Estensis III G 6 (ef. ib.; in Quaestionibus idem mihi E erat G). contulit de fato librum Schwartz.

Vaticanus Urbinas 54 (cf. p. XXII) contuli ipse. K

HavNiensis BVH 88 Fabr. forma octava, constat foliis 104 adglutina- $H$ tis impressae Pselli in sex philosophiae modos introductioni; continet post philosophi argumenti nonnulla excerpta fol. 57-59 Alexandri de casu, 59-64 de fato disputatiunculas ex libri De anima mantissa, fol. 64-94 librum De fato ad imperatores, 94-104 excerpta ex Quaestionibus II, 1-16 (cf. Ch. Graux Not. somm. des ms. gr. de Copenhague Paris 1879 p. 104). contuli ipse.

Parisinus 1868, miscellaneus, continet fol. $82^{v}$ sq.; librum De A fato inde a verbis àmapáßacóv $\tau \iota v \alpha$ 166,2 usque ad finem. contulit Iulius Koch. 
C Parisinus 2049 continet post Themistii in Aristotelis De anima libros paraphrasin totum libellum De fato. partes contulerunt Immisch et Iulius Koch.

Parisinus suppl. 292. continet inde a fol. $79^{\mathrm{r}}$ totum libellum, sed $79^{r}$ et $v$ initium usque ad verba àmapáßatóv $\tau \imath v \alpha$ (excl.) „ex Aldino impresso transseriptum ", ut in margine legitur. sequitur $80^{\mathrm{r}}$ idem quod in cod. 1868 inest, praemissis his verbis „Alexandri Aphrodisiensis (antea

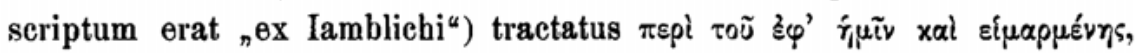
cui deest initium in volumine 683 bibliothecae regiae, ibi incipit verbis

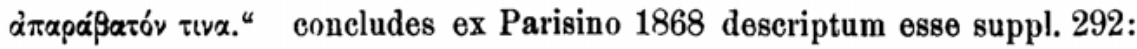
et removetur omnis dubitatio eo, quod in cod. 1868 leguntur pristinae computationis vestigia 683 et 2647 . de his rebus edocuerunt me Immisch et Corssen. neglexi igitur hune codicem.

Fragmentum denique continet libelli, i. e. initium usque ad verba

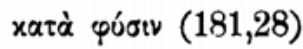

D Vossianus 25 miscellaneus fol. 149-163 (cf. Cat. Bibl. publ. Leyd. p. 396). contuli ipse.

T Bernensis 402 miscellaneus fol. 127-137 (ef. Hagen catalogus librorum Bernensium p. 365). partem olim contulit Usenerus et mecum benigne communicavit.

$a^{1} a^{2} \quad$ Accedunt ad novem hos libros Trincavelli editiones duae, Aldinae officinae prior anni 1534 '), altera anni 1536 Zanettianae.

Haec omnia non exstarent, nisi unus Venetus 258 Alexandri librum servasset. quod ut intellegamus, non opus est difficiliore argumentatione. Eusebins testis est primum 166,28. 29 et 168,18, ubi Veneti lacunas unus Eusebius supplet, libri omnes aeque hiant. Eusebium autem, quod in transeursu moneam, dolendum est, ea, quae ex hoc libello excerpsit, non ad verbum descripsisse. diligenter tamen omnia ex Gaisfordiana editione adnotavi, ex quibus videmus eum certe in posterioribus Alexandri verba libere immutasse. quod autem 167,24 et 168,6 cum Eusebio $a^{2}$ et recentiorum

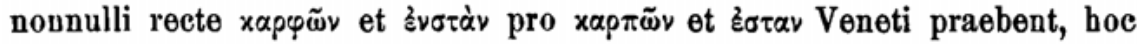
puto coniectura factum esse. sed redeo ad propositum. ubicumque igitur gravius laborat Veneti textus, aegrotare etiam apographa consentaneum est,

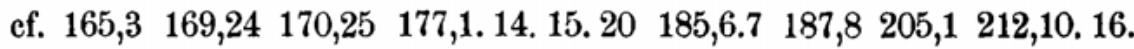
differentiarum autem, quae in eis obveniunt, praeter vitia et coniecturas

1) Omnia Themistii opera. Alexandri Aphr. libri duo De anima et De fato unus Venetiis 1534 (secundo folio Trincavelli epistola ad Contarenum legitur). 
sequioris temporis explicationem optimam subministrant Veneti V correcturae, quas librarii pro suo quisque arbitrio modo secuti sunt modo sprevere. hoc observavi e. c. $166,23 \quad 171,25 \quad 177,13 \quad 180,10 \quad 184,24 \quad 186,1$ 187,21 194,4. 14 196,18-27.28.29 199,22. $25 \quad 202,2 \quad 205,23$. sed hoc mihi credas condonesque exactam harum minutiarum tabulam.

Iam vero in duas classes sevocantur apographa lacunis, qua laceri sunt 179,5-6 codices ACKDTa'a ${ }^{2}$ (item 204,4-5), integri mansere BHES. deinde in hac altera familia suum locum obtinent ES, qui et ES multis peculiaribus lacunis turbati sunt, et Veneti quasdam proprietates

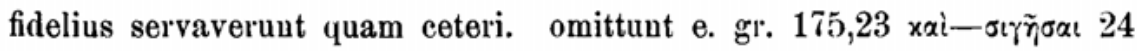

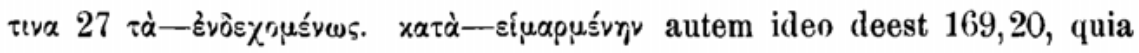
baec verba $\mathrm{m}^{2}$ margini adscripsit. ita soli ES retinuere $\pi \alpha^{\lambda} \lambda_{\iota \nu} 172,28$. ceterum

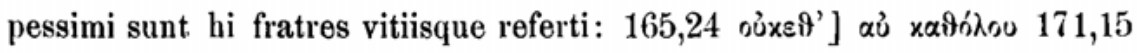

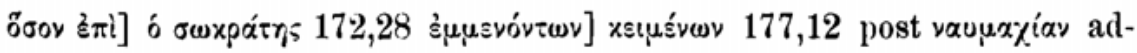

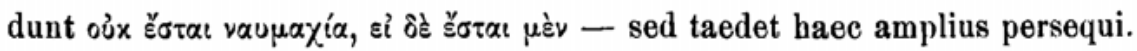

Etiam alterius familiae certa sunt genera $\mathrm{ACDTa}^{1}$ et $\mathrm{Ka}^{2}$ et inter illa primum prorsus conspirare $\mathrm{A}$ et $\mathrm{C}$ notaverim. ita omittunt $166,12 \mathrm{AC}$

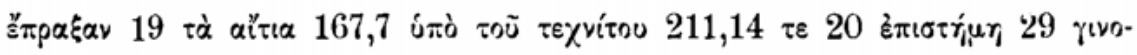

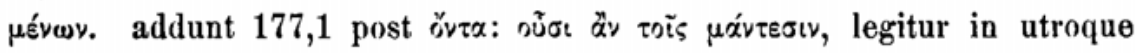

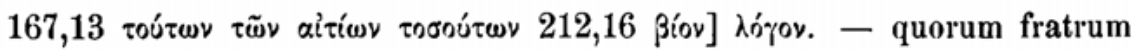

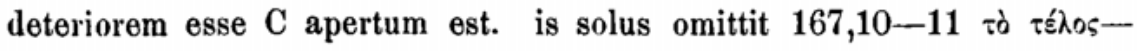
จะò่ $201,12-13$ siitaque neglego hune post 166,2; nam initium libri in C solo exstat.

Aldinae porro cum codice A similitudo est maxima. tamen neque ex $\mathrm{Aa}^{1}$ boc impressa est, neque hic ex illa descriptus. evitavit enim A haec Aldinae $\begin{array}{lllllllllll}\text { vitia } & 164,8 & 166,14 & 167,17 & 169,15 & 170,21 & 171,2 & 173,4.11 & 176,1.16 & 179,12\end{array}$ $\begin{array}{llllllll}180,12 & 182,2 & 183,9.19 & 187,25 & 189,3.28 & 190,9.19 .20 & 193,5.9 & 194,11\end{array}$ 196,27 200,22 203,2 205,20. 28 206,2 207,9 209,18 210,14. 18 211,15.

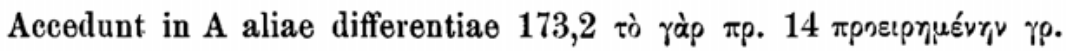

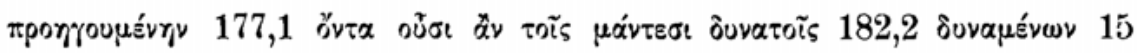

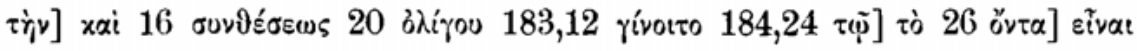

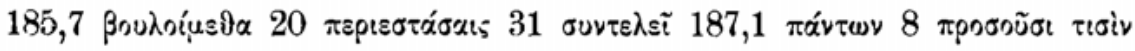

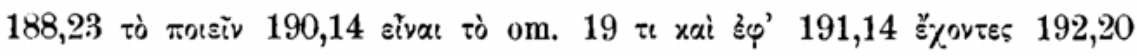

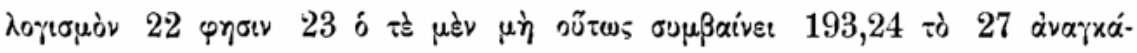

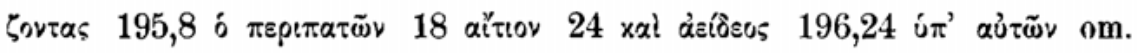

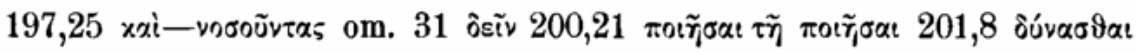

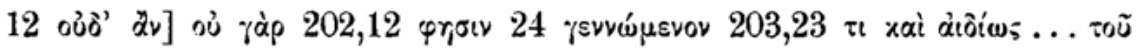

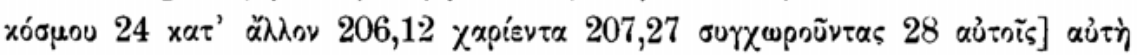




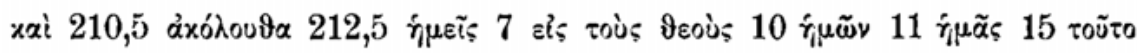

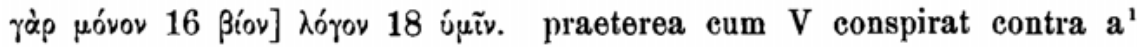
179,12. 14. 23 186,27 188,13 192,19 193,4 194,24. adnota denique de-

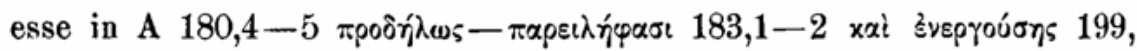

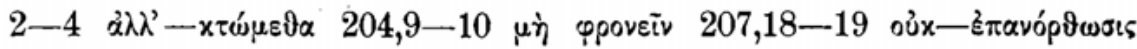

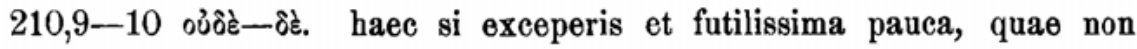
commemoravi, sit tibi $\mathbf{a}^{1}$ instar codicis $\mathrm{A}$, ex cuius gemello accuratius confecto Aldinam provenisse patet.

DT Denique ad eundem fontem, ex quo manaverunt $\mathrm{ACa}^{1}$, redit etiam DT, quos geminos esse inde primum colligitur, quod in utroque fragmen-

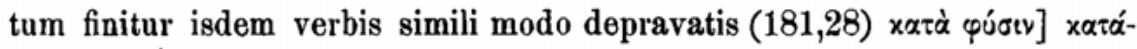

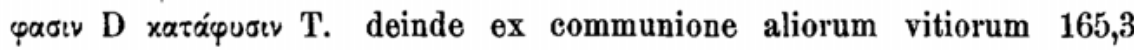

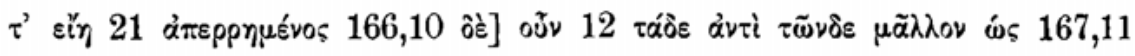

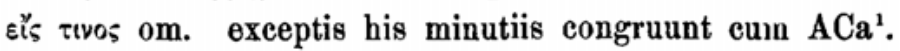

$\mathrm{Ka}^{2} \quad$ Vinculo vel artiore, quam A cum $\mathrm{a}^{1}$, coniuncta est altera editio $\left(\mathrm{a}^{2}\right)$ cum codice Vaticano $\mathrm{K}$, de quo supra dixi. editione hic liber peior est, nam

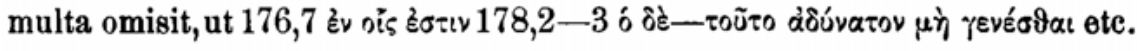
tamen quominus apographum eum esse putemus Aldinae, impediunt editio-

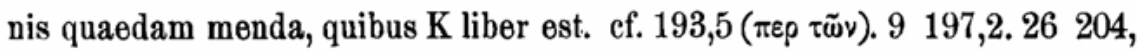

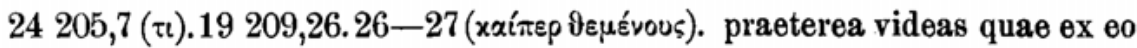
adnotavi 188,13 192,23.24 198,14.15 206,23 210,7. sed haec ideo tantum adscribo, ut hune codicem nunc plane neglegam. itaque tantum de editione loquar, in qua animadvertimus aliquam factam esse cum certa alterius familiae stirpe permixtionem. sunt enim in $a^{2}$ lectiones diversae $a b a^{1}$, quas invenimus etiam in ES. huius generis sunt, ut ex multis pauca eligam

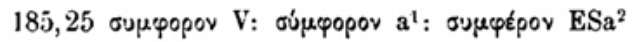

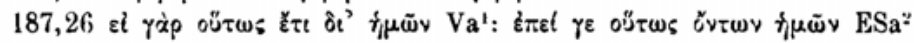

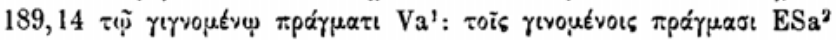

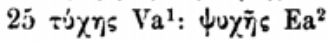

$191,2 \beta$ los add. $\mathrm{Ea}^{2}$

et simile est 176,11 , de quo loco inspicias adnotationem meam.

Quare videntur primum propria via e Veneto $\mathrm{V}$ emanasse archetypi et editionum et eius familiae, cuius nos depravatos tantum prognatos ES novimus. in conficiendo autem eo codice, ex quo Zanettiana impressa est, haec ipsa familia adhibita est, euius auctorem non sine iudicio Venetum olim exscripsisse testantur etiamnum paucae lectiones, quas potui sequi codicum ES: 170,26 171,4 202,26.

$a^{1} a^{2} \quad$ Sequitur ut Editiones inter se conferam. quas primum satis magna copia deturpat mendorum communium. alia deinde alterutri propria 
sunt, etsi omissionibus parcius peccat $a^{2}$. quae quia in apparatu omnia proposui, hic praetereo. sed duo moneo. primum, discriminis quod intercedit inter duas editiones magnam partem ex Veneti correcturis profectam esse, ef. velim 166,18.19. $23 \quad 169,24 \quad 171,25 \quad 172,13.19 .25$ 173,24 ete. deinde, iam in eo codice, ad quem $a^{1}$ et $a^{2}$ quasi ad communem matrem redeunt, vestigia apparere artis eriticae. nam in utroque recte mutatum est e. gr. $173,9 \quad 182,16 \quad 186,19 \quad 189,16 \quad 193,16.17$ falso $176,6177,15$. denique singulae editiones suum passae sunt redactorem, cuius rarius in $\mathrm{a}^{1}$ obveniunt conamina, neque tamen desunt, velut $165,3167,8168,3178,10.11$. plura invenies in $a^{2} 165,26167,24168$, 6. 14 173,10. 15. $26178,1+189,27 \quad 191,2.24 .25200,16201,8$ ef. praeterea 165,9. 23 168,19. $21 \quad 169,22 \quad 177,9$ 187,8. $26 \quad 201,10202,10203,17$.

Restat ut Veneti 261 et Hauniensis rationem habeamus. quod de B illo p. XIX dixi, valet etiam hic. editori inutilis est, sed memoratu digna omnia, quae $\mathrm{B}^{2}$ adnotavit. Hauniensis autem primo obtutu videtur directa via descendisse ex Veneto $\mathrm{V}$, cuius et varias lectiones iterat et

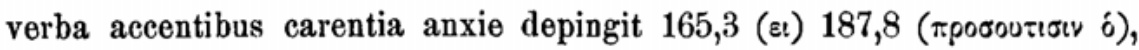
veteris correctoris monita sequitur, ubi $a^{1}$ ot $a^{2}$ ea uegleguni 180,10

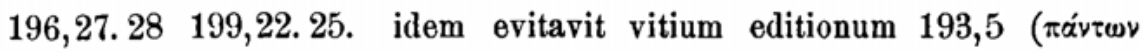
$\tau \tilde{\omega} \nu \mathrm{H}$ ) et neglegere solet, quae illae novaverunt: $176,6 \quad 177,15 \quad 182,16$ 187,26 191,24. 25 193,16.17. sed consentit $\mathrm{H}^{1}$ cum $\mathrm{a}^{2} 191,2$ cum $\mathrm{a}^{1}$ 170,26 cum E 186,30 189,16 192,20 195,26 (ẃs) 196,16 198,25. maxima porro eorum pars, quae margini adscripsit librarius Hauniensis, ex E provenit 167,25 (ópla E) 170,25 173,15 181,11 183,17. 32 184,1. 2187 , 13. 24 192,18. postremo et in margine et in textu lectiones iuveniuntur quae ex libris mihi notis non desumptae sunt: 165,21 169,24.30 171,5.6 $176,18185,20186,19189,12 \quad 193,3 \quad 196,29$ 197,13. videntur igitur huius libri auctori praeter fidele Veneti apographum (nam ipsum Venetum non exscripsit of. 194,4) cum alii tum eius familiae codex praesto fuisse, quam nos libris ES novimns.

Habes librorum catalogum, quo patebit cur Veneti lectiones cum pulvisculo exhauserim, ex apographis meo arbitrio mutuatus quae mihi digna viderentur. licebat autem eo parciorem esse, quia veterum editionum discrepantia ex consilio harum editionum accurate adnotata, quid litterae renascentes Alexandro cum profuerint tum nocuerint optime docet.

Bagolini versionem, quam ille primum edidit Venetiis cum Quaestionibus anno 1541, in apparatum non recepi. nam etsi certum librum inter eos quos supra examinavi non licet indicare, quem ille interpretatus 
sit, dubitatione tamen exemptum est, praeter Veneti $\mathrm{V}$ apographa nibil ei suppeditasse. ne Eusebio quidem usus est, nam vertit 166,28-29

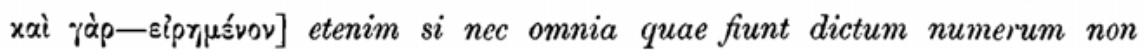
excedunt et 167,24 ubi verum vel ex $\mathrm{a}^{2}$ potuit discere cum Veneto fructuum quorundam attrectationes. dubitantibus hanc tabulam propono locorum in Veneto corruptorum et ad verbum a Bagolinis redditorum

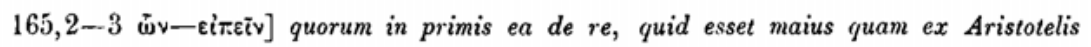
sententia dicere

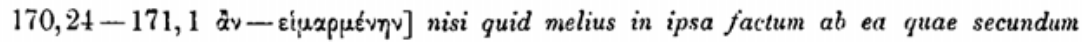
naturam emoverit, dolores sustinebunt, et mala patientur et in talibus exitus rursus vitae secundum fatum sunt

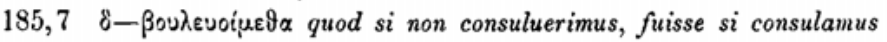

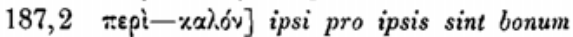

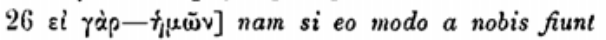

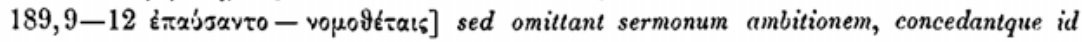
quod in nobis solutum esse liberumque arbitrium et auctorem in eligendis oppositisque agendis, in his quae hominibus occurrunt merito versari creditum, tum a vulgaribus quam a legislatoribus

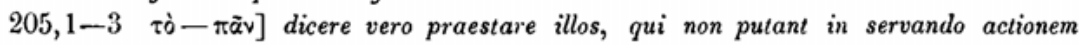
aninalium impulsoriam servari iam et id, quod in nobis, eo quod non omne.

Addo ipsum emblema, quod 165,24 casu irrepsit sensu plane carens, ab illo servatum esse. ex neutra editionum pendet Bagolinus, etsi cum utraque affinitate coniunctus est. consentit enim cum posteriore 177,20 $187,8 \quad 189,25 \quad 191,2 \quad 201,10$, cum utraque $182,16 \quad 186,19 \quad 189,16 \quad 199,22.25$ ignorat autem posterioris emendationem $191,24.25$ et prioris 165,3 ; lacunas, quae $\mathrm{a}^{1}$ et $\mathrm{a}^{2}$ communes sunt, explevit, et cum codice $\mathrm{A}$ et $\mathrm{C}$ consentit

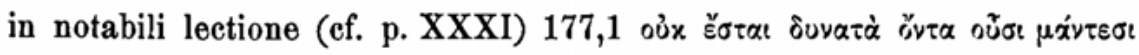

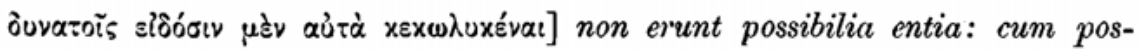
sint ipsi vates, qui quidem cognoscunt, ea ipsa impedivisse. liber igitur sive libri, quibus Bagolini usi sunt, intermedii iacent inter Venetum et editiones.

Ediderunt libellum post Trincavellum Ioannes CASELIUS Rostochii anno 1588, Anonymus cum latina versione Londini 1658, Ioannes ORelli cum Hugonis Grotii versione latina Turici 1824.

CASELIUS quibus subsidiis usus sit, in praefatione, quo Andream PaULLIUM adloquitur, „quocum ante duo et viginti annos in eodem contubernio Bononiae satis familiariter vixit ${ }^{\mu}$, et tacet neque nos cum difficultate quaerere oportet. nam Zanettianae editioni, cuius et vitia et omissiones repetit, tam arte se adplicavit, ut dubitare liceat, num Aldinam noverit. ei autem posterioris editionis exemplo, quo usus est, videntur ex libris manuscriptis, quos supra recensui, nonnullae lectiones adscriptae fuisse. ita explicatur e. gr. 196,16 209,26.27. 
Ut Caselio Zanettiana, ita Axonymo Aldina editionis fundamentum fuit. praeterea autem et illa ei nota fuit et Eusebius, ut in praefatione ipse fatetur: quae in Aldinis exemplaribus Graecis truncata sunt aut quovis morlo mutilata ex Euscbio partim ex coniectura non vana restituimus et versionem perspicuam magis quam concinnam adiecimus. et supplevit quidem ex Eusebio 166,28-29, lacunas autem quas ex altera editione sarcire potuit hiantes reliquit. non desunt emendandi conamina Caselii audaciora, velut $177,1.2 .5$ 180,16.

OrelLn editioni maxime profuit quod Hauniensis collatione uti potuit; neque Caspari Orellii symbolis, quibus librum ornavit, sine fructu utere. ipse autem nec recensendi nec emendandi muneri par id quod ingenio peccavit ne diligentia quidem compensavit. nam adnotatio erroribus scatet.

Post Bagolinos elegantissime librum latine interpretatus est Hugo Grotrvs in libro Philosophorum veterum de fato et de eo quod in nostia est potestate collectae partim et de graeco versae, Parisiis 1624; Lugduni Batarorum 1648. 1679. germanice Schvltess in libro Bibliothek der griechischen Philosophen T. İV p. 150 sq., gallice Nourisson in libro De la liberté et du hasard Paris 1870.

Chrysippea ex Alexandri libro collegit et recensuit A. GERCKE in Supplementis annalium philologorum anni 1885 p. 691 sqq. nonnullos locos tractavit Heine in programmate "Stoicorum de fato doctrina" Naumburgi 1855 . 